\title{
Criminologie
}

\section{Quand recherche et savoir scientifique cèdent le pas à l'activisme et au parti pris}

\section{Ezzat A. Fattah et Rabia Mzouji}

Volume 43, numéro 2, automne 2010

Les 50 ans de l'École de criminologie : aperçu de la recherche d'ici et d'ailleurs

URI : https://id.erudit.org/iderudit/1001770ar

DOI : https://doi.org/10.7202/1001770ar

Aller au sommaire du numéro

\section{Éditeur(s)}

Les Presses de l’Université de Montréal

ISSN

0316-0041 (imprimé)

1492-1367 (numérique)

Découvrir la revue

Citer cet article

Fattah, E. A. \& Mzouji, R. (2010). Quand recherche et savoir scientifique cèdent le pas à l'activisme et au parti pris. Criminologie, 43(2), 49-88.

https://doi.org/10.7202/1001770ar
Résumé de l'article

Populaires à la naissance de la victimologie, les études individuelles de victimes de crimes spécifiques ont été éclipsées, dans les années 1970, par des enquêtes de victimisation à grande échelle, l'approche micro cédant alors la place à une approche macro. Le but premier de ces enquêtes consistait à déterminer le volume de victimisation, à identifier la population de victimes, ainsi qu'à établir leurs caractéristiques sociodémographiques. Chacune de ces enquêtes donne une mine d'informations sur les victimes et permet une analyse minutieuse des modèles et des tendances spatiotemporelles pour des types variés de victimisation.

Lors des trois dernières décennies $\mathrm{du} \mathrm{xx}^{\mathrm{e}}$ siècle, cependant, la victimologie $\mathrm{a}$ subi une métamorphose très importante mais aussi inquiétante. La transformation idéologique de la victimologie a été nuisible à l'enrichissement et au progrès de la victimologie scientifique. La mutation idéologique de la victimologie est manifeste dans les conférences et les symposia qui se tiennent en son nom : l'étude des victimes qui cède le pas à l'art de les aider, la sur-identification avec des victimes de crimes, le zèle du missionnaire avec lequel les « intérêts » de la victime sont défendus et poursuivis, etc. Tout cela signale un glissement préoccupant : de savoir scientifique dépassionné, non biaisé et impartial, la victimologie est devenue un plaidoyer politique tombé dans un sectarisme déclaré. Le zèle du missionnaire montré par de nombreux victimologues au nom et dans l'intérêt des victimes de crimes est lourd de dangers. Quelques-uns de ces dangers sont examinés dans le présent article.
Ce document est protégé par la loi sur le droit d'auteur. L’utilisation des services d’Érudit (y compris la reproduction) est assujettie à sa politique d'utilisation que vous pouvez consulter en ligne.

https://apropos.erudit.org/fr/usagers/politique-dutilisation/ 


\title{
Quand recherche et savoir scientifique cèdent le pas à l'activisme et au parti pris
}

\author{
Ezzat A. Fattah \\ Professeur émérite \\ École de criminologie, Université Simon Fraser \\ fattah@sfu.ca
}

RÉSUmé - Populaires à la naissance de la victimologie, les études individuelles de victimes de crimes spécifiques ont été éclipsées, dans les années 1970, par des enquêtes de victimisation à grande échelle, l'approche micro cédant alors la place à une approche macro. Le but premier de ces enquêtes consistait à déterminer le volume de victimisation, à identifier la population de victimes, ainsi qu'à établir leurs caractéristiques sociodémographiques. Chacune de ces enquêtes donne une mine d'informations sur les victimes et permet une analyse minutieuse des modèles et des tendances spatiotemporelles pour des types variés de victimisation.

Lors des trois dernières décennies $d u x x^{e}$ siècle, cependant, la victimologie a subi une métamorphose très importante mais aussi inquiétante. La transformation idéologique de la victimologie a été nuisible à l'enrichissement et au progrès de la victimologie scientifique. La mutation idéologique de la victimologie est manifeste dans les conférences et les symposia qui se tiennent en son nom: l'étude des victimes qui cède le pas à l'art de les aider, la sur-identification avec des victimes de crimes, le zèle du missionnaire avec lequel les "intérêts» de la victime sont défendus et poursuivis, etc. Tout cela signale un glissement préoccupant: de savoir scientifique dépassionné, non biaisé et impartial, la victimologie est devenue un plaidoyer politique tombé dans un sectarisme déclaré. Le zèle du missionnaire montré par de nombreux victimologues au nom et dans l'intérêt des victimes de crimes est lourd de dangers. Quelques-uns de ces dangers sont examinés dans le présent article.

MOTS-CLÉS - Victimologie, victimologie activiste, victimisation, enquêtes de victimisation, victimisation confrontationnelle, victime catalyseuse, victime récidiviste.

Traduit de l'anglais par Rabia Mzouji.

Criminologie, vol. $43, \mathrm{n}^{\circ} 2(2010)$ 


\section{Première partie : les débuts de la victimologie}

Une brève histoire de la victimologie ${ }^{1}$

On associe généralement l'émergence de la victimologie comme champ de recherche universitaire à la publication, en 1948, de l'ouvrage précurseur de Hans von Hentig, The Criminal and his Victim, aux Presses universitaires de Yale.

Le terme de victimologie a été lui-même forgé en 1949 par un psychiatre étatsunien, Frederick Wertham, qui l'a utilisé pour la première fois dans son livre The Show of Violence, livre dans lequel il insistait sur la nécessité d'ériger la victimologie en science.

Devenue pourtant populaire, la victimologie attend toujours que son histoire approfondie soit retracée et écrite. Il n'existe pas davantage d'évaluations systématisées de sa situation actuelle ni de ses probables destinées.

La victimologie est une discipline jeune et prometteuse, elle reste un sujet fascinant. Et bien que la victimisation soit aussi vieille que l'humanité elle-même, ce n'est qu'au sortir de la Deuxième Guerre mondiale que l'étude scientifique des victimes de crimes a émergé comme complément essentiel à la recherche criminologique déjà bien établie sur les agresseurs. Parce que la victimologie a émergé pour combler un sérieux vide théorique, elle n'a pas tardé à faire partie intégrante de la criminologie. Et bien qu'elle se soit alors affirmée comme un champ de recherche majeur de la criminologie, sa nature, son importance et son statut continuent à générer beaucoup de commentaires et de controverses. Quoi qu'il en soit, l'étude des victimes de crimes et celle de la victimisation criminelle ont le potentiel pour complètement remodeler la criminologie et pourraient même annoncer une rupture paradigmatique depuis longtemps attendue, tant la criminologie en a désespérément besoin. Comme cette dernière, la victimologie n'a pas suivi le même chemin dans toutes les parties du globe. Et comme bien d'autres disciplines, elle est plus avancée et développée dans certains pays que dans d'autres. Et s'il y a certains points communs et similarités dans la manière dont la victimologie s'est bâtie ici et là, il y a aussi des différences qualitatives et même quantitatives significatives. Quoi qu'il en soit, l'évolution récente de la victimologie a été à la fois considérable et dramatique. La discipline a en effet subi une transformation radicale.

1. Pour une histoire plus détaillée, voir Fattah (1967a, 1967b, 1971, 1978 et 1991). 
Les approches théoriques qui ont caractérisé la première victimologie ont été éclipsées par des réalisations majeures dans le champ appliqué. Cette phase remarquable dans l'évolution de la victimologie a conduit à sa consolidation, à la collecte de données, à la formulation de théories et, par-dessus tout, à de nouvelles législations en matière de victimes, à des efforts soutenus pour améliorer leur condition, mais aussi pour soulager leur détresse. Sur le plan théorique, des modèles variés ont été élaborés pour tenter d'expliquer les variations énormes dans les risques de victimisation, pour mieux saisir le regroupement de victimisations dans certains lieux et pour certains groupes, ou encore pour dénouer le phénomène intrigant de la victimisation répétée. Sur le front législatif, il y a eu une multitude de projets de loi en matière de victimes dans un grand nombre de pays. En suivant la Déclaration des principes fondamentaux de justice pour les victimes de criminalité et pour les victimes d'abus de pouvoir adoptée par l'Assemblée générale des Nations Unies en 1985, les déclarations des droits des victimes ont été adoptées par les législatures de plusieurs pays. Les avancées dans le champ appliqué ont même été plus spectaculaires encore. Parmi celles-ci, citons la création d'une compensation étatique pour les victimes de crimes violents, le retour à une restitution demandée à l'agresseur, ou encore la mise sur pied et la prolifération de programmes de médiation victime-agresseur. Les services d'aide aux victimes ont eux aussi connu une grande expansion. Quant à la thérapie des victimes, elle est devenue la façon commune et «dans l'air du temps» de traiter les effets traumatisants de la victimisation. Si la thérapie victimologique a été encouragée et mise en place dans certaines cultures, elle a été désapprouvée dans d'autres. Tout cela a conduit à promouvoir une connaissance et des programmes en victimologie. Des cours et des séminaires en victimologie existent maintenant depuis plusieurs décennies dans certaines universités mais restent totalement absents dans d'autres. De telles différences ne devraient toutefois pas rendre impossible la tâche de dresser un portrait plus ou moins unifié de l'évolution de la discipline ou encore une analyse perspicace de son état actuel et de ses aménagements futurs. Même si on dispose à la fois de vues d'ensemble globales et d'informations très détaillées, les généralisations et les simplifications restent inévitables. 


\section{La promesse initiale de la victimologie théorique}

Le premier traitement systématique des victimes de crimes a vu le jour en 1948 dans le livre de Hans von Hentig The Criminal and his Victim. Dans la quatrième partie du livre, sous le titre provocant «La contribution de la victime à la genèse du crime» (notre traduction), il critiquait l'étude statique et unidimensionnelle de l'agresseur qui avait dominé la criminologie jusque-là. À la place, il suggérait une nouvelle approche, dynamique et dyadique, qui accorde autant d'attention à la victime qu'au criminel. Von Hentig avait déjà traité le sujet plus tôt dans un article publié en 1940/1941 dans le Journal of Criminal Law and Criminology. Il y indiquait que:

C'est vrai que beaucoup d'actes criminels ont peu ou n'ont pas du tout de rapport avec la personne agressée. [...] D'un autre côté, nous pouvons fréquemment observer une interdépendance réelle entre l'auteur et la victime, le tueur et la personne tuée, le dupeur et la personne dupée. Bien que cette opération réciproque soit un des phénomènes les plus curieux de la vie criminelle, il a échappé à l'attention de la socio-pathologie. (Von Hentig, 1940/1941 : 303; notre traduction)

Dans son livre, von Hentig notait en outre que:

La loi considère certains résultats et les derniers gestes qui y conduisent. Elle fait ici une distinction claire entre la personne qui agit et la personne qui souffre. En observant la genèse d'une situation, dans un nombre considérable de cas, nous rencontrons une victime qui consent tacitement, coopère, conspire ou provoque. La victime est un des facteurs de causalité. (Ibid.: 436; notre traduction)

Von Hentig a insisté sur le fait que de nombreuses victimes contribuaient à leur propre victimisation, soit en incitant ou en provoquant le criminel, soit en créant ou en encourageant une situation davantage susceptible de conduire à la commission du crime. D'autres pionniers en victimologie, qui croyaient fermement que les victimes peuvent consciemment ou pas jouer un rôle causal dans ce qu'elles subissent, ont souligné les diverses formes que leur contribution pouvait prendre: négligence, insouciance, témérité, imprudence, etc. Ils ont noté que le rôle de la victime pouvait être motivationnel (attirer, éveiller, induire, inciter, aguicher, etc.) ou fonctionnel (provoquer, précipiter, déclencher, faciliter, participer, etc.) (Fattah, 1991). Le livre de von Hentig a été suivi d'études théoriques qui traitaient des types de victimes, des relations victime-agresseur, et du rôle joué par les victimes dans certains 
types de crimes. Le livre a donné aussi un élan à plusieurs recherches empiriques qui portaient une attention particulière aux victimes de délits spécifiques comme l'homicide (Wolfgang, 1958; Fattah 1971), le viol (Amir, 1971), le vol (Normandeau, 1968), les coups et blessures (Pittman et Handy, 1964; Curtis, 1974), les offenses de pédophilie (Virkkunen, 1975), la fraude (Padowetz, 1954), le chantage (Hepworth, 1975). Durant les premières années de la victimologie, la littérature sur les victimes de crimes restait relativement modeste si on la comparait à celle en criminologie. Dans les années 1980, toutefois, une grande vague de livres et d'articles importants a marqué une nouvelle ère de la victimologie (Rock, 1994). Aujourd'hui, il n'est pas exagéré de dire que l'étude des victimes de crimes est devenue une part intégrante de la criminologie. Sa contribution à cette discipline est aussi évidente qu'axiomatique. Il peut donc sembler surprenant que la nécessité d'un tel apport ait échappé à l'attention des criminologues pendant plus d'un siècle. Mais il n'est pas rare, en sciences sociales, d'occulter ce qui est pourtant le plus visible. Ce point a été bien saisi par Rock (1994) qui note:

Même la criminologie et la sociologie de la déviance - à savoir, les disciplines qui se concentraient le plus directement sur l'analyse du crime, les criminels et la justice pénale - ont eu tendance d'une manière ou d'une autre à occulter la victime pendant un long moment, échouant à voir ce qui, rétrospectivement, aurait probablement dû sauter aux yeux. De telles omissions arrivent continuellement. Elles sont la part inéluctable de toute discipline, la conséquence d'une vérité qu'on doit à Burke quand il disait qu'«une manière de voir est toujours une manière de ne pas voir». Le prix qui consiste à organiser, spécialiser et accumuler du savoir à propos d'une certaine aire est l'abandon systématique d'autres matières, expulsées du noyau dur et envoyées au-delà des marges. Précisément parce que la criminologie est une discipline tournée vers l'empirie, elle a eu tendance à ignorer ces choses qui ne portent pas le nom de crime, de criminel et de justice pénale. (Rock, 1994: 11; notre traduction)

Bien que la victimologie soit maintenant fermement établie comme un champ d'étude majeur au sein de la criminologie, sa teneur, sa portée et son statut continuent, nous l'avons dit, à susciter beaucoup de débats et de querelles. Rock (1994) décrit la victimologie comme une «discipline relativement amorphe». Et au cinquième Symposium international de victimologie (Zagreb, août 1985), Cressey a même ouvertement déclaré que la victimologie n'était ni une discipline scientifique ni un champ académique (voir plus loin). 
Populaires à la naissance de la victimologie, les études individuelles de victimes de crimes spécifiques ont été éclipsées, dans les années 1970, par des enquêtes de victimisation à grande échelle, l'approche micro cédant alors la place à une approche macro. Le but premier de ces enquêtes consistait à déterminer le volume de victimisation, à identifier la population de victimes, ainsi qu'à établir les caractéristiques sociodémographiques des victimes de crimes. Si cette approche macro se montra suffisamment riche pour étudier les tendances et les modèles en victimisation ainsi que pour analyser la distribution sociale et spatiale de certains types de crimes, elle restait par contre assez limitée en ce qui concerne les circonstances sociales et personnelles dans lesquelles ces crimes avaient pris place. Cette approche peinait à comprendre les dynamiques psychologiques et sociales du comportement criminel, le processus de sélection de la victime, les interactions victime-agresseur, le rôle dynamique de la victime dans les divers crimes, etc. La victimologie des débuts était essentiellement théorique, elle se concentrait presque exclusivement sur les explications causales du crime et sur le rôle de la victime dans ces explications. Elle mettait essentiellement l'accent sur les caractéristiques des victimes, leurs relations et autres interactions avec leurs «victimiseurs», et l'analyse du comportement de la victime comme variable situationnelle, comme facteur déclenchant, précipitant ou actualisant. Ce cadre théorique, proposé par von Hentig, a guidé la recherche qui a fait école et qui a été entre autres portée par Ellenberger, Wolfgang, Amir, Normandeau, Curtis, Silverman et Fattah (voir plus haut). La prise en compte de la détresse des victimes de crimes a d'abord pu être trouvée dans les modestes programmes étatiques de compensation pour ces victimes dans certains pays comme la Nouvelle-Zélande, l'Angleterre, le Canada ou les États-Unis. La redécouverte des victimes de crimes, menée par le mouvement féministe, un mouvement qui s'est fait le champion de la cause des victimes de viols, d'agressions sexuelles et de violences domestiques, a généré beaucoup d'empathie et de sympathie pour un groupe en grande partie privé de ses droits (Fattah, 1978, 1994a).

\section{La victimologie et ses premières escarmouches}

La victimologie théorique est devenue l'objet d'attaques injustifiées et de critiques idéologiques non fondées. Certains l'ont définie comme l'«art de blâmer la victime» (Clark et Lewis, 1977). La victimologie a 
pris alors une nouvelle forme: aider et assister les victimes de crimes, soulager leur détresse et affirmer leurs droits. Un mouvement politique est né et la victimologie a été de plus en plus définie et reconnue à travers sa composante appliquée. Les rencontres de victimologie reflétaient les transformations qu'elle opérait: passage d'une discipline scientifique à un mouvement humaniste, glissement de la recherche savante vers l'activisme politique. Ces rencontres étaient souvent transformées en plaidoyers au nom des victimes.

\section{La controverse autour du concept de «victime catalyseuse»}

Un des concepts les plus vilipendés et les plus controversés en victimologie est celui de «victime catalyseuse» («Victim Precipitation»). La critique est toutefois, du moins pour l'essentiel, injustifiée. Il faut revenir ici à l'échec des critiques à saisir la distinction subtile entre concepts disculpatoires et concepts explicatifs. Si cette distinction est bien comprise, il devient clair que le concept de «victime catalyseuse» n'est rien d'autre qu'une tentative académique légitime qui tente de comprendre les motivations du crime, d'analyser les dynamiques des interactions victime-agresseur et d'expliquer la chaîne d'événements qui ont ultimement conduit à l'acte de victimisation. Dans un tel modèle explicatif, il n'est aucunement question de position normative ou de jugement de valeur tels la culpabilité ou le blâme. Quoi qu'il en soit, les critiqueurs du concept de «victime catalyseuse» n'ont, dès le départ, pas accepté ce pour quoi le concept avait été forgé ni ce qu'il pouvait bien signifier, mais insistaient plutôt sur le fait qu'il avait été forgé et utilisé pour blâmer la victime. Certaines critiques allèrent même plus loin encore. Plutôt que limiter leurs attaques au seul concept de «victime catalyseuse», ils étendirent leur critique à la discipline victimologique au complet. Un bon exemple d'une telle posture se retrouve dans la citation suivante de Clarke et Lewis (1977):

Dans les sciences sociales, blâmer la victime est devenu une rationalisation de plus en plus populaire pour les comportements «déviants» et criminels. [...] Ces dernières années, blâmer la victime a été institutionnalisé dans le monde universitaire sous la forme de la victimologie. [...] Le chercheur masculin trouve son échappatoire dans la victimologie. Il cherche la cause du problème dans le comportement de la victime et va se convaincre (et le public avec lui) qu'en changeant ce comportement, le problème peut être sous contrôle. La victimologie devient alors l'art de blâmer la victime. (Clarke et Lewis, 1977: 147, 148, 150; notre traduction) 
Les biais idéologiques et de genre sous-tendant les critiques ci-dessus sont beaucoup trop évidents pour nécessiter un argument de rejet. Ce qui est par contre plus surprenant, c'est le fait que les féministes attaquent le concept de «victime catalyseuse» tout en expliquant (et même en justifiant) que si des femmes finissent par recourir à la violence face à leurs maris ou à leurs amants agressifs, c'est à la suite des raclées et autres mauvais traitements que ces derniers leur ont infligés! Les cas d'homicide, les tentatives de meurtre et les agressions ont été invariablement expliqués en référence au comportement préalablement violent de la victime masculine. Le «syndrome de la femme battue» est devenu une défense acceptée devant les tribunaux de juridiction criminelle. Ce que les féministes ont fait, c'est donc recourir à la notion de «victime catalyseuse» sans la nommer comme telle et apparemment sans se rendre compte que cette position était contradictoire avec leur posture initiale à propos de ce concept.

Sans entrer davantage dans les détails des débats qui ont entouré le concept de «victime catalyseuse», qu'il suffise de noter qu'il n'est pas vrai, comme certaines critiques (Franklin II et Franklin, 1976) l'ont affirmé, que ce concept réduit l'agresseur à un acteur passif qui ne se met en action qu'en réponse au comportement de la victime. Ce qui est vrai, c'est que ce concept est une forme de comportement manifeste, agressif et provocant de la victime qui sert de déclic à l'action de l'agresseur. Comme tel, c'est simplement un facteur actualisant, le stimulus qui suscite la réponse violente. Ce qui pourrait être vu comme une «action» si elle était regardée unilatéralement sera ainsi interprété comme une «réaction» ou une «réaction excessive» (selon les circonstances du cas à l'étude) quand elle sera lue dans un modèle dynamique interactionniste des interrelations entre victime et agresseur. Recourir au concept de «victime catalyseuse» comme variable explicative, c'est maintenir que s'il n'y avait pas eu d'actions précipitantes de la victime, la victimisation contre cet individu particulier n'aurait pas pris la forme qu'elle a prise dans cette situation particulière (Fattah, 2004).

\section{Les résultats surprenants et inattendus des enquêtes de victimisation}

Une des premières tâches de la victimologie théorique consiste à compiler les données empiriques sur les victimes de crimes. Le principal outil utilisé jusqu'à présent pour compiler cette information, ce sont les 
enquêtes de victimisation, qu'elles soient menées à l'échelon local, régional, national ou international. On peut retenir de ces enquêtes celles qui sont réalisées sur une base régulière et à intervalles réguliers en Angleterre et aux États-Unis: la British Crime Survey et la National Crime Survey. Chacune de ces enquêtes donne une mine d'informations sur les victimes de crimes. Toutes deux permettent une analyse minutieuse des modèles et des tendances spatiotemporelles pour des types variés de victimisation. L'objectif initial de ces enquêtes, qui visait pour l'essentiel à comptabiliser les victimisations, a été largement étendu. Ces dernières années, plusieurs nouvelles questions ont été ajoutées à l'outil de recherche afin d'explorer des zones non couvertes par le passé comme les paliers de peur du crime, les échelons de satisfaction avec l'action policière, les raisons de ne pas rapporter un incident à la police, les conséquences de la victimisation, etc. Les enquêtes examinent, en outre, les mesures prises par les répondants pour empêcher certains types de délits, ou pour minimiser les risques d'une future victimisation. Certaines enquêtes ont essayé d'établir quel lien pouvait exister entre l'agression et la victimisation et ce, en incluant des questions demandant aux répondants d'auto-rapporter les actes de délinquance qu'ils avaient pu commettre. Ces dernières questions ont révélé une forte interrelation entre agression et victimisation. Dans leur enquête à Londres, en Angleterre, Sparks et al. (1977) ont découvert que les victimes de crimes violents avaient significativement plus de chances que les non-victimes d'auto-rapporter la commission de crimes violents. Gottfredson (1984) a analysé les données de l'enquête britannique sur le crime de 1982 et a été frappé par l'interrelation relativement forte entre le fait d'être agresseur et le fait d'être victime. Pour les personnes qui (auto)rapportaient au moins un délit violent, la probabilité d'une victimisation personnelle était de $42 \%$, soit sept fois plus que pour des personnes n'ayant (auto-)rapporté aucun délit violent.

L'enquête britannique sur le crime concernant l'Écosse (Chambers et Tombs, 1984) a révélé que $40 \%$ des répondants ayant admis un acte d'agression étaient eux-mêmes victimes d'agressions durant la même période de temps. En dépit des problèmes méthodologiques et pratiques des enquêtes de victimisation, et en dépit de leurs limites, elles ont permis aux chercheurs de compiler une somme considérable de données sur les victimes de crimes qui est extrêmement riche en variété et en détail. Grâce aux enquêtes de victimisation, nous savons maintenant que la criminalité et la victimisation sont concentrées sur certains 
groupes et dans certaines zones, et qu'il y a beaucoup plus d'affinités entre agresseurs et victimes que ce qui est communément admis. Il ne s'agit pas de dire ici que toutes les victimes de crimes partagent les caractéristiques de leurs agresseurs. Mais plutôt de seulement insister sur le fait que ces deux populations ont plusieurs caractéristiques en commun. Que ce soit en Europe, aux États-Unis ou en Australie, la recherche a montré que les agresseurs impliqués dans les types de crimes couverts par les enquêtes de victimisation sont, de façon disproportionnée, des hommes jeunes, résidents urbains, au statut socioéconomique bas, au chômage (sans être à l'école) et célibataires. Concernant les États-Unis, on peut également ajouter une surreprésentation des Noirs. Quant aux enquêtes de victimisation, elles ont révélé que les victimes partagent, elles aussi de façon disproportionnée, ces caractéristiques. Elles ont donc confirmé que les profils démographiques des victimes de crimes et des criminels condamnés sont remarquablement similaires (Gottfredson, 1984).

Plusieurs chercheurs (Hindelang et al., 1978; Singer, 1981) ont découvert, en particulier pour les crimes d'agression, que victimes et auteurs se rejoignaient non seulement dans leurs caractéristiques démographiques mais aussi dans leur façon de percevoir des situations de menace physique ou psychologique. On comprend que la fréquence avec laquelle certains individus deviennent impliqués dans des situations enclines à la violence affectera à la fois leurs chances de recourir à la violence et d'en être les victimes, d'attaquer et d'être attaqué, de blesser et d'être blessé, de tuer et d'être tué. Finir par être une victime ou par être légalement considéré comme agresseur dépend, assez souvent, de facteurs dus au hasard plutôt que d'une action délibérée, d'une planification ou d'une intention. Si les rôles victime/agresseur ne sont pas nécessairement antagoniques, ils sont fréquemment complémentaires et interchangeables (Fattah, 1994b).

Une étape importante sur la route d'une victimologie comparative a été atteinte avec les enquêtes internationales sur le crime. Les enquêtes constituaient une tentative utile pour compiler des données de victimisation qui étaient standardisées par l'adoption d'un même questionnaire dans plusieurs pays. L'objectif principal de ces enquêtes était d'éviter des problèmes liés au fait de comparer des données qui auraient été compilées au moyen d'outils différents utilisant des méthodologies qui le seraient tout autant. Les données de terrain pour la première enquête internationale sur le crime ont été recueillies en janvier 1989. L'enquête 
s'est appuyée sur l'interview téléphonique assistée par ordinateur (ITAO) et les résultats ont été publiés en 1990 (Van Dijk et al., 1990).

L'enquête internationale sur le crime a ensuite lancé une deuxième salve en 1992. Certains des pays qui ont participé à la première enquête, tels que la Suisse, la Norvège et l'Irlande du Nord, n'ont pas pris part à la deuxième. Mais la deuxième enquête incluait certains pays d'Europe de l'Est qui n'avaient pas participé à la première, comme la Pologne ou l'ancienne Tchécoslovaquie (voir Del Frate et al., 1993).

Le troisième balayage de l'enquête internationale sur les victimes de crimes a été accompli en 1996-1997, dans vingt pays en transition. Il y avait d'anciens pays socialistes de l'Europe de l'Est (à l'est, de la Pologne à la Mongolie; au sud, de l'Albanie et de la Bulgarie à la Macédoine; et au nord, jusqu'aux pays baltes, l'Estonie, la Lettonie et la Lituanie). Les rapports nationaux tirés de cette enquête ont été publiés en 1998 par l'Institut interrégional de recherche des Nations Unies sur la criminalité et la justice (UNICRI) (Hatalak et al., 1998).

La quatrième vague a été réalisée en 2000 dans 47 pays. Elle a été suivie par la cinquième (2004/5). La sixième a eu lieu en 2009. En dépit de la prolifération d'enquêtes de victimisation et de leur utilité incontestable, ce qu'elles mesurent exactement n'est pas encore clair. Leurs objectifs à long terme sont eux aussi mal établis. La victimisation est une expérience relativement culturelle, subjective et individuelle (Fattah, 1993b). Le sentiment d'avoir été victime ne coïncide pas toujours avec la définition légale de victimisation. Que tentent dès lors exactement de mesurer les enquêtes de victimisation? Leur objectif est-il de compter les victimisations criminelles qui répondent aux critères établis par le Code criminel, ou s'agit-il plutôt de mesurer les expériences subjectives de victimisation des répondants? Il va sans dire que nous parlons là de deux réalités différentes. Les enquêtes sont-elles faites pour mesurer le crime ou la victimisation? Les titres «enquête sur le crime» et "enquête de victimisation» continuent à être utilisés de façon interchangeable (Fattah, 1997) et les récentes enquêtes internationales ont été appelées, nous l'avons dit, «enquêtes internationales sur les victimes de crimes» (The International Crime Victim survey). La mine d'informations essentiellement recueillies par l'intermédiaire d'enquêtes de victimisation a mené à des formulations théoriques diverses. Des modèles ont été développés pour offrir des explications plausibles aux variations dans les risques de victimisation, mais aussi pour éclairer le regroupement de victimisation dans certaines zones et 
pour certains groupes. Ils ont aussi aidé à dénouer le phénomène intrigant de la victimisation répétée. Les différents modèles sont présentés et synthétisés dans mon livre Understanding Criminal Victimization (Fattah, 1991).

\section{Deuxième partie : certains enjeux importants encore négligés dans la recherche sur les victimes}

\section{Le recours à la victime comme agent de neutralisation et de légitimation}

Lattitude du «victimiseur» envers la victime est un domaine important en victimologie, mais qui n'a reçu qu'une attention limitée de la part des chercheurs. Dans de nombreux cas, l'attitude de l'agresseur envers la victime est caractérisée par l'apathie et l'indifférence. Un autre aspect intéressant à relever provient du fait que les délinquants semblent nourrir des stéréotypes sur leurs probables victimes. La plupart ne choisissent pas leurs victimes au hasard mais les sélectionnent attentivement et, durant ce processus de sélection, font des distinctions subtiles entre cibles illégitimes et cibles légitimes, cibles appropriées et cibles inappropriées, etc. Les attitudes des "victimiseurs», les images mentales et les stéréotypes des victimes potentielles jouent un rôle important dans le processus de raisonnement précédant la victimisation (Fattah, 1976).

Quand on analyse le processus précédant la victimisation, on peut identifier et distinguer différents processus mentaux: neutralisation, redéfinition/autolégitimation et désensibilisation. La neutralisation est centrée sur le «victimiseur». Son objectif principal consiste à rendre ce dernier capable de surmonter les barrières culturelles et morales qui associeraient l'acte commis à une victimisation. La redéfinition/autolégitimation met l'accent sur l'acte de victimisation. Son but essentiel vise à redéfinir, à rationaliser et à justifier l'acte. Le troisième processus, la désensibilisation, s'oriente sur la victime future. Sa visée première consiste à désensibiliser le «victimiseur» à la douleur et aux souffrances qu'il infligera à la victime. Si le processus de neutralisation opère de façon à rendre les mécanismes de contrôle social inefficaces, le processus de redéfinition conduit donc à dépouiller l'acte de son caractère délinquant, illégal et immoral. Quant au processus de désensibilisation, il doit permettre à l'agresseur d'infliger des blessures physiques et 
morales à la victime sans éprouver un malaise ou un sentiment de culpabilité, sans souffrir d'une dissonance cognitive post-victimisation (Fattah, 1991).

Les «victimiseurs» disposent de techniques variées de désensibilisation. Parmi ces techniques, on retrouve le déni de la victime, sa réification, sa désindividualisation, sa dépersonnalisation, ou encore la dénégation des blessures qui lui sont infligées. À ces techniques s'ajoutent celles de blâmer la victime, de la dévaloriser, de la déshumaniser, etc. Blâmer la victime est une des techniques les plus populaires de neutralisation et de désensibilisation. En recourant à cette technique, le «victimiseur» est à même de se convaincre lui-même que la victime lui a fait du mal, à lui ou à d'autres proches de lui, et qu'il y a par conséquent une injustice à réparer. Une fois que la culpabilité de la victime, réelle ou imaginée, est établie dans l'esprit de l'agresseur, toute compassion pour la victime et tout sentiment de culpabilité personnelle peuvent être écartés. On devrait insister ici sur le fait que si blâmer la victime est une technique banale et populaire largement utilisée, ce n'est pas pour autant un processus de distorsion intentionnelle. Dans la plupart des cas, le «victimiseur» est en fait complètement convaincu de la culpabilité de la victime et perçoit son passage à l'acte comme un acte de justice vengeresse. Les techniques de désensibilisation mentionnées ci-dessus ont bénéficié d'un très important support empirique dans la recherche menée par les psychologues sociaux (Fattah, 1991).

Les sentiments d'injustice, le fait ou l'impression d'avoir été victimisé sont aussi des mécanismes importants dans les processus de neutralisation aussi bien que dans les processus de redéfinition/ autolégitimation et de désensibilisation. Ce n'est pas surprenant, par conséquent, qu'un grand nombre de délinquants et de criminels se perçoivent eux-mêmes plus ou moins comme des victimes. Ce fait explique le phénomène d'interchangeabilité de la victime et du «victimiseur» (voir plus loin) et pourquoi de nombreuses victimes se transforment en agresseurs. Les enfants abusés qui deviennent plus tard des abuseurs d'enfants, des populations opprimées qui deviennent des oppresseurs sans pitié, des victimes d'injustices politiques qui deviennent des terroristes dénués de culpabilité sont simplement quelques exemples de cette métamorphose. 


\section{Les rôles interchangeables de la victime et du victimiseur}

Quand notre approche pour aider les victimes n'est pas fondée sur la science mais sur un plan politique et idéologique, elle est fondamentalement erronée. Le processus actuel consistant à identifier qui est victime mais aussi à décider qui peut solliciter les services, l'assistance et les compensations aux victimes est à la fois simpliste et arbitraire. Un seul incident est en effet utilisé pour assigner un statut de victime. Un statut qui non seulement octroie des droits et privilèges à la personne qui en bénéficie mais qui détermine aussi qui est la victime et qui est l'agresseur, ignorant ainsi les dynamiques situationnelles et comportementales du processus de victimisation. Dans des situations en effervescence et changeant rapidement telles que les altercations, les disputes, les querelles, les bagarres, etc., la partie blessée est quasi automatiquement définie comme la victime sans égard à la question de savoir si oui ou non elle était l'instigatrice, l'initiatrice, l'assaillante initiale, etc. Comme la plupart des crimes de violence sont le plus souvent des actes de vengeance, le résultat final est un indicateur très pauvre pour identifier la réelle victime. Les représailles sont un ingrédient clé dans la violence (Felson et Steadman, 1983) et la vengeance est la motivation la plus courante dans l'usage de la force. La violence gratuite est l'exception, pas la règle. Dans la plupart des cas rencontrés, la violence est l'expression d'une rancune, la réponse à une attaque, une blessure ou une provocation. L'approche actuelle ignore le fait que les rôles de la victime et de l'agresseur ne sont ni statiques, ni fixés, ni assignés. Ils sont dynamiques, complémentaires et interchangeables, la même personne se déplaçant successivement, voire simultanément, entre les deux rôles. Quand l'agression fait face à l'agression, quand la violence répond à la violence, les rôles sont simplement renversés. L'agresseur initial devient la victime et la victime initiale finit par être définie comme l'agresseur. Tout ce que cela suggère, c'est que l'agression et la victimisation ne sont pas deux phénomènes opposés mais bien les deux faces de la même médaille. Ce sont des événements reliés et parallèles dans l'expérience de vie de nombreux individus (Fattah, 1994). Cela montre aussi qu'à la différence des perceptions populaires (ou plutôt des perceptions erronées), les populations de victimes et d'agresseurs ne sont pas, comme on le croit communément, deux populations distinctes et mutuellement exclusives. Elles sont en fait des populations homogènes qui se chevauchent dans une grande mesure. 
Les victimes d'hier sont souvent les agresseurs d'aujourd'hui, et les agresseurs d'aujourd'hui sont fréquemment les victimes de demain. La popularité du modèle prédateur/proie est telle que beaucoup refusent de même entretenir l'idée que l'agression et la victimisation pourraient ne pas être des phénomènes opposés et mutuellement exclusifs, mais bien des événements reliés et parallèles dans la vie de nombreux individus. Pour ceux qui se font les champions de la cause des victimes de crimes et qui font campagne en leur nom, l'idée que pour beaucoup de gens la vie est un cycle continu de victimisations et d'agressions est une idée fantaisiste. Ils ne croient pas davantage crédible d'aller à l'encontre de la dichotomie populaire entre l'agresseur actif et la victime passive, l'agresseur coupable et la victime innocente, les bons Abel et les maléfiques Caïn. L'erreur du modèle prédateur/proie peut être facilement détectée quand on voit des enfants sexuellement abusés devenir euxmêmes des abuseurs d'enfants à l'âge adulte. L'erreur apparaît aussi de façon plutôt évidente dans le cas des jeunes de la rue qui passent constamment d'un rôle à l'autre, parfois en quelques minutes, parfois en quelques heures. À cause de leur expérience quotidienne, il est impossible de leur coller l'étiquette de délinquant ou de victime, ou encore de les classer dans une seule de ces deux catégories. Pour ces jeunes, la violence et même la mort sont une part intégrante de leurs expériences visuelles quotidiennes. Elles constituent un fait banal de leur vie. Leur existence quotidienne est une chaîne de délinquances et de victimisations. Ils s'engagent dans des activités délinquantes, illégales et à haut risque pour survivre et sont, de façon répétée, victimisés physiquement, sexuellement et financièrement par d'autres jeunes plus âgés et plus aguerris à la rue. Dans certains pays d'Amérique du Sud ou d'Amérique centrale, ils sont même exécutés par les célèbres escadrons de la mort (Fattah, 1994 et 1997).

\section{La réponse de la victime à la victimisation} "confrontationnelle» et son effet sur le résultat final

L'étude des relations victime-agresseur qui a reçu beaucoup d'attention dans le premier âge de la victimologie cède en ce moment le pas à l'étude des dynamiques de victimisation et des interactions victimeagresseur ainsi qu'à leur potentiel résultat. Une analyse en profondeur de ces résultats est essentielle pour comprendre les dynamiques de la victimisation violente (et de plusieurs types de victimisation non 
violente). Cette approche interactionniste situationnelle analyse divers types de victimisation criminelle non pas comme des comportements unilatéraux, mais comme des transactions situationnelles. C'est le cas des victimisations de face-à-face où une interaction brève ou prolongée a lieu entre la victime et le «victimiseur». Dans de telles situations, la réponse de la victime à une victimisation non voulue et habituellement inattendue est, dans une large mesure, non préméditée et non planifiée. La spontanéité de la réaction est sans aucun doute partiellement responsable des variations extrêmes des réponses des victimes à des situations identiques et ce, alors même que les expériences de victimisation semblent très similaires. La variété des réponses des victimes est, en retour, responsable des différents résultats qui émergent de ces victimisations «confrontationnelles». Les résultats sont aussi déterminés ou conditionnés par un grand nombre de variables. Les caractéristiques sociodémographiques des victimes sont, par exemple, fortement associées à la probabilité de subir une blessure. Le risque de blessure varie aussi par type de délit et, pour des raisons plutôt évidentes, est plus élevé dans le cas d'un viol que dans celui d'un vol qualifié, pour des voies de fait graves que pour des voies de fait simples (Fattah, 1984).

Les études qui se penchent sur la réponse de la victime dans une victimisation de face-à-face restent à un stade embryonnaire et doivent encore résoudre nombre de problèmes conceptuels et méthodologiques. Il serait nécessaire, entre autres, d'élaborer une typologie de réponses et de construire les instruments adéquats pour mesurer les niveaux de résistance. Un problème important concernant les données a trait à la séquence temporelle des résistances et des réponses, en particulier à la difficulté d'établir si c'est l'usage de la force par l'agresseur qui a précédé la résistance de la victime ou si c'est l'inverse.

Il y a aussi un besoin urgent d'étudier minutieusement les diverses corrélations des réponses de victimes lors d'une confrontation de faceà-face. Ces corrélations touchent entre autres à l'âge, au sexe et à l'origine ethnique de la victime; la présence ou non d'une arme; la relation victime-agresseur; l'heure et le lieu de l'attaque; l'élément de surprise; le nombre de victimes et d'agresseurs impliqués dans la situation; l'homogénéité/l'hétérogénéité sur le plan ethnique et du genre; la différence d'âge entre l'agresseur et la victime; la présence d'alcool et/ou de drogues; et la présence d'autres personnes non impliquées dans la situation de victimisation (Fattah, 1991 : 216). 
Inutile de dire qu'il y a un lien causal direct entre la résistance au crime et sa commission. La recherche empirique montre que la résistance de la victime est la variable la plus fortement associée à l'achèvement ou le non-achèvement du délit. Il y a aussi des preuves empiriques indiquant une association positive entre, d'une part, la résistance et, d'autre part, la fréquence et la gravité des blessures dont souffrent les victimes (voir par exemple Block, 1977 ; Zimring, 1977; Wright, 1980; Wolfgang, 1982; Ziegenhagen, 1985 ; Block et Skogan, 1986; Chappell et James, 1986; Cook, 1986; Zimring et Zuehl, 1986; U.S. Bureau of Justice Statistics, 1987). Il faudrait évidemment bâtir une théorie de la réponse de la victime à la victimisation «confrontationnelle». Une telle théorie aurait des implications significatives pour réduire le risque de mort et de blessures parmi les victimes de viol, de vol qualifié, de voies de fait, d'enlèvement, etc. Elle constituerait un socle pour réfléchir aux conseils à donner aux victimes potentielles sur la meilleure façon de réagir quand elles se trouvent en situation de victimisation de face-àface. Une autre tâche de la recherche consisterait à élaborer une typologie de réponses et à établir la fréquence, les déterminants et les corrélations de chacune d'elles. En lien avec ces objectifs, il faudrait clairement définir ce qu'on veut dire par «efficacité» et établir les critères selon lesquels l'efficacité des différents types de réaction pourrait être mesurée.

Alors que la plupart des interactions et des confrontations victimeagresseur sont marquées par la rage, la colère, l'hostilité et les antagonismes, on a observé que dans certains cas d'enlèvement, de prise d'otage et de détournement d'avion, quelques victimes réagissent par une réponse émotionnelle positive plutôt que négative à l'égard de leurs ravisseurs. On ne comprend pas encore très bien ce qui conduit à ces liens affectifs inattendus. Pour tenter d'expliquer leur apparition, deux syndromes ont été avancés: le syndrome de Stockholm et le syndrome du kidnappé (bijackee syndrom) (voir Fattah, 1980).

\section{La sélection de la victime/cible et ses implications pour la prévention de la victimisation}

Les enquêtes de victimisation ont montré que les risques et les taux de victimisation criminelle ne sont pas également distribués dans la population générale. Cela suggère que les victimes/cibles de crimes ne sont pas choisies au hasard, que certaines victimes/cibles sont préférées à 
d'autres, que certaines sont souvent choisies là où d'autres sont évitées. Ce chapitre négligé de la victimologie a des implications théoriques et pratiques très importantes. Il faut par conséquent d'urgence mener plus de recherches sur ce que les délinquants (professionnels, occasionnels et amateurs) regardent quand ils cherchent une victime, voir à quels critères ils recourent quand ils sélectionnent une cible parmi beaucoup d'autres.

Bien que les critères de sélection soient divers et varient d'un délinquant à l'autre, et bien que «l'attrait» et «l'adéquation» de la cible soient des concepts relatifs et subjectifs, le peu de recherches qui ont été menées sur la sélection de cibles nous permet d'identifier et de classer un grand nombre de facteurs susceptibles de contribuer et de conduire au choix du délinquant.

Les facteurs les plus souvent sélectionnés peuvent être regroupés sous cinq titres: 1) proximité; 2) attrait ; 3) accessibilité ; 4) manœuvrabilité; et 5) risque (Fattah, 1991).

La proximité est importante parce que la plupart des agresseurs ne traversent pas de longues distances pour commettre leurs crimes. Leur recherche d'une victime/cible adéquate est généralement confinée à l'espace qu'ils connaissent. La bonne connaissance de l'espace, aussi bien que la familiarité avec la victime/cible, est susceptible, d'une part, d'accroître le sentiment de l'agresseur qu'il agit en zone de confort et de sécurité et, d'autre part, d'augmenter sa confiance.

Naturellement, l'attrait de la victime/cible varie selon les délits et les délinquants. On y retrouve des facteurs tels que les caractéristiques personnelles de la victime: l'attrait physique (en particulier, pour les crimes sexuels), la rentabilité/lucrativité (pour les crimes commis pour un gain financier), la vulnérabilité, l'«adéquation»/«concordance», etc.

L'accessibilité de la victime/cible, à la fois temporelle et géographique/spatiale, est aussi un facteur de sélection important. La localisation géographique, le site, la disposition des lieux et la facilité d'accès à la victime/cible constituent certaines de ces caractéristiques auxquelles une importance peut être accordée par le délinquant quand il choisit parmi plusieurs cibles «disponibles».

La manœuvrabilité de la victime est elle aussi susceptible d'être un facteur important dans les crimes où il y a une confrontation de faceà-face entre protagonistes tels que lors d'un viol, une agression sexuelle, un vol, une agression, un enlèvement, une prise d'otage, etc. En portant leur attention sur une victime ou une cible en particulier, les agresseurs 
sont plus à même d'évaluer leur capacité à contrôler la situation, à contraindre la victime et à générer ce qu'ils en attendent.

Il y a enfin une foule de facteurs de risque que les agresseurs potentiels prennent en considération quand ils choisissent entre plusieurs victimes/cibles. Ces facteurs incluent le niveau et le type de protection de la cible (voire son absence de protection); le degré de surveillance de la cible; les dangers inhérents à l'attaque de la cible, tels que la probabilité que la victime soit armée; la probabilité que l'agresseur soit blessé ou tué par la victime; la probabilité d'être interrompu par une tierce partie ou par la police; la probabilité d'une intervention policière; l'estimation de l'agresseur quant aux potentielles sanctions s'il est arrêté et condamné (Fattah, 1991: 253).

La vulnérabilité de la victime et la victimisation répétée: la victime récidiviste

Depuis les débuts de la criminologie, les criminologues ont essayé d'expliquer le comportement criminel en référence aux caractéristiques personnelles de l'agresseur. De la même façon, les victimologues ont essayé d'expliquer la victimisation, en particulier la victimisation répétée, en référence aux caractéristiques personnelles et sociales de ceux qui sont victimisés. L'élan qui a été donné à une telle recherche peut être retracé dans la preuve indiscutable que les risques de victimisation criminelle ne sont pas également distribués dans la population générale et que les victimes de crimes ne constituent pas un échantillon représentatif et exempt de biais de cette population. Les enquêtes de victimisation ont montré sans l'ombre d'un doute que la probabilité et les taux réels de la victimisation criminelle sont liés aux caractéristiques personnelles des victimes et varient selon certaines variables sociodémographiques. Elles ont aussi révélé que les victimes de crimes souffrent de manière disproportionnée d'une variété d'autres infortunes. Tout cela suggère une certaine vulnérabilité ou prédisposition à la victimisation. Cette vulnérabilité ou cette prédisposition ne devraient pas être interprétées comme insinuant une sorte de fatalisme ou d'inévitabilité. Elles sont similaires à la notion de "diathèse», fréquemment rencontrée en médecine et qui suggère que certains individus peuvent être davantage à risque et plus susceptibles d'attraper certaines maladies que d'autres. En victimologie, la vulnérabilité et la prédisposition sont utilisées pour expliquer les variations dans le risque et dans les probabilités statisti- 
ques de victimisation. Il doit être clairement dit que les variations des risques ne signifient pas que ces derniers ont pour causes certaines caractéristiques personnelles comme la maladresse ou l'incongruité. Ces variations sont bien plus probablement le résultat de différences sur le plan de la situation sociale, des aires de résidence, des occupations, de l'exposition et de l'accessibilité, etc. Bien que les notions de vulnérabilité et de prédisposition soient parfois utilisées de façon interchangeable, elles ne sont pas des concepts identiques. La vulnérabilité n'est qu'une dimension de la prédisposition et en aucun cas, sa dimension la plus importante (Fattah, 1991).

Il est possible d'identifier divers types de prédispositions: spatiale, structurelle, liée à la déviance, professionnelle, situationnelle, etc. Ces types sont suggérés par des variations dans les risques de victimisation. Les citadins, par exemple, courent un plus grand risque de victimisation criminelle que les gens de la campagne, et dans une grande ville, les gens vivant ou travaillant dans certaines zones sont plus enclins à la victimisation que d'autres. En outre, la victimisation criminelle ne se regroupe pas que spatialement, elle se concentre aussi socialement. Cela suggère ce qui peut être formulé comme une «prédisposition structurelle». Cette prédisposition peut être retracée en fonction de l'âge, du genre (féminin), du statut (minoritaire), du chômage, etc. Les styles de vie déviants conduisent à une prédisposition à la victimisation. C'est le cas, par exemple, des usagers et des trafiquants de drogues, des homosexuels et des prostituées. L'étiquetage négatif, la stigmatisation sociale, le manque ou l'absence de protection, la nature des activités dans lesquelles la personne s'engage et les conditions dans lesquelles ces activités ont lieu sont des facteurs importants qui conduisent les membres de ces groupes à une prédisposition à la victimisation. La prostitution par exemple est une activité à risque extrêmement élevé. Elle rend les prostituées, en particulier les prostituées de la rue, extrêmement vulnérables à divers types de victimisation criminelle (Fattah, 2002 et 2003).

Certains types de prédispositions sont temporaires et transitoires et peuvent par conséquent être considérés comme des états de «vulnérabilité éphémère», par exemple un état de vulnérabilité chimique qui résulte d'une consommation d'alcool. L'alcool est un important facteur victimogène et peut contribuer de diverses manières au risque de victimisation. Ce constat est confirmé par un corpus croissant de preuves empiriques montrant un haut pourcentage de victimes de violence ayant consommé de l'alcool dans les heures précédant leur victimisation (Fattah, 1991). 


\section{Victimes récidivistes et chroniques}

Quand on utilise la fréquence de victimisation comme critère, les victimes de crimes peuvent être divisées en groupes: celles pour lesquelles cette situation n'arrive qu'une fois, les occasionnelles; et celles pour lesquelles la victimisation est chronique, les récidivistes. L'étiquette de «victime récidiviste» peut être utilisée pour décrire un individu, un commerce ou une organisation qui est victimisé fréquemment ou de façon répétée, à des occasions différentes et dans un espace de temps relativement court, que ce soit par le même agresseur ou par plusieurs. Quand des victimisations fréquentes, répétitives et du même type surviennent à la même personne et se prolongent sur une longue période de temps, cette personne peut endosser le statut de «victime chronique».

Il y a des recherches empiriques qui soutiennent l'existence de victimes à la fois récidivistes et chroniques. Le psychiatre allemand Reimer Hinrichs (1987) a utilisé l'étiquette «victime chronique» comme titre d'un livre dans lequel il rapporte les découvertes d'une recherche comparative sur la victimisation qu'il a menée à Berlin-Ouest et à Philadelphie.

Les victimes récidivistes et chroniques ont une valeur particulière pour la recherche victimologique. Elles peuvent aider à comprendre les facteurs et les variables associés à certains types de victimisation criminelle et peuvent enrichir notre compréhension des notions de prédisposition et de vulnérabilité (Fattah, 1991).

\section{Troisième partie: de la victimologie de l'acte à la victimologie de l'action}

\section{La flétrissure de la victimologie théorique}

Dans les années 1960, Sutherland et Cressey (1960: 55) soutenaient que Lombroso et l'École positive italienne avaient retardé de cinquante ans le progrès de la recherche sur l'étiologie du crime en le considérant comme un phénomène individuel plutôt que social, en contraste avec des écoles précédentes (pensons à Guerry et Quetelet). Une critique similaire pourrait être faite aux victimologues qui ont retardé le progrès de la victimologie théorique en transformant une jeune discipline scientifique attrayante et prometteuse en champ de bataille idéologique. La critique féroce qu'ils ont adressée à la recherche théorique en victimologie a eu un effet intimidant sur les pionniers de la victimologie 
qui ont cessé d'étudier la responsabilité et le rôle fonctionnel de la victime de peur d'être accusés de se positionner pour les agresseurs et contre les victimes. Des études prometteuses sont alors devenues presque des sujets tabous: l'analyse des événements criminels comme interactions ou transactions situées, l'étude du comportement de la victime comme variable situationnelle, l'analyse de la «victime catalyseuse» et d'autres formes de contribution des victimes à la genèse du crime. Comment les chercheurs pouvaient-ils agir autrement quand ils lisaient Timmer et Norman (1984: 66) et affirmer que «l'idéologie de la "victime catalyseuse" ne blâme ni la structure de la société ni l'agresseur individuel pour le crime commis. À la place, elle blâme la victime qui en précipiterait la commission»? De façon frappante, leur critique était similaire à ce que Franklin II et Franklin (1976) avaient affirmé presque une décennie plus tôt, quand ils avaient argumenté que la «victime catalyseuse» réduit l'agresseur à un acteur passif qui n'est mis en action que par le comportement de la victime. Il est aisé de voir que ce qui sous-tend ces critiques tient pour l'essentiel à une interprétation erronée, en particulier quand cette interprétation affirme que toute démarche entreprise pour expliquer la victimisation à un niveau micro - en référence au comportement de la victime - serait en fait une tentative délibérée de blâmer la victime et de mettre l'accent sur les causes individuelles plutôt que sur les causes structurelles du crime. L'erreur de cette assertion est claire. La victimologie ne cherche pas à expliquer le crime mais à expliquer la victimisation. Elle ne cherche pas à expliquer pourquoi des gens deviennent criminels mais pourquoi des gens (les cibles) deviennent victimes et d'autres pas. Évidemment, une telle démarche ne peut pas être faite sans examiner les caractéristiques, le comportement et le style de vie de celles et ceux qui sont victimisés. L'affirmation qu'agir ainsi détourne des causes structurelles du crime est injustifiée. Expliquer les risques différentiels ainsi que les variations des taux réels de victimisation exige une analyse en profondeur, non seulement des caractéristiques individuelles de la victime, mais aussi des facteurs structurels qui facilitent la vulnérabilité et la prédisposition tels que l'âge, le genre, le statut minoritaire, le chômage, la pauvreté, etc. La recherche théorique en victimologie tente d'aider à comprendre le rôle que les facteurs structurels jouent dans l'étiologie de la victimisation. Les macro-explications nécessitent toutefois d'être enrichies par d'autres explications, à même d'expliquer la victimisation dans des cas individuels: pourquoi cette victime/cible particulière a-t-elle été choi- 
sie? Pourquoi la victimisation est-elle arrivée dans cette situation spécifique, à ce moment spécifique et dans ce lieu spécifique, et dans les circonstances dans lesquelles elle a eu lieu? De là, l'intérêt de mieux comprendre et de davantage parfaire l'opérationnalisation de concepts tels que la vulnérabilité de la victime, la "victime catalyseuse», la participation de la victime, son imprudence, sa négligence, etc.

Malheureusement, les critiques véhémentes contre le concept de «victime catalyseuse» ont exercé un effet intimidant sur les chercheurs qui n'osaient plus parler ni du rôle ni de la contribution de la victime à la genèse du crime. Un exemple assez frappant de cette intimidation se trouve dans l'étude de Gibson et al. (1980) sur le viol au Manitoba, dans laquelle les auteurs proposent ce qu'ils appellent «une théorie situationnelle du viol». Ils critiquent les explications "génétiques» du viol et démontrent aisément la supériorité des explications qui tiennent compte des facteurs situationnels. En rapportant les résultats de leur recherche et en exposant leur théorie, les auteurs parlent des temporalités qui facilitent le viol (facilitating times), des lieux qui facilitent le viol (facilitating places), des circonstances qui facilitent le viol (facilitating circumstances), du matériel qui facilite le viol (facilitating hardware), des acteurs qui facilitent le viol (facilitating actors). Il est amusant de noter que les auteurs n'osent pas parler des victimes qui facilitent le viol! Quand ils discutent le rôle de la victime, le mot victime est remplacé par le mot «autres» (facilitating others)! Ils n'acceptent pas la définition d'Amir du «viol catalysé par la victime». Mais au lieu d'élaborer leur propre définition et d'appliquer cette définition aux cas étudiés, ils emploient celle utilisée par Wolfgang pour les cas d'homicide pour conclure qu'i1 n'y a pas de cas dans leur matériel qui peuvent être qualifiés de viol précipité par la victime. Ils écrivent:

Dans son étude sur l'homicide à Philadelphie, Wolfgang définissait les «homicides catalysés par la victime» (victim precipitated homicides) comme ceux pour lesquels la victime avait été la première à recourir à la force physique lors de l'altercation qui avait ultimement conduit à sa mort. Si le viol est vu comme une agression forcée, dans les termes de Wolfgang, un "viol catalysé par la victime» renverrait à une situation dans laquelle la victime a été la première à introduire un élément de coercition physique. Si on suit cette définition, aucun des viols inclus dans cette étude ne pourrait être qualifié de «viol catalysé par la victime». (Gibson et al., 1980: 51; notre traduction) 
Après avoir rejeté la définition donnée à la «victime catalyseuse» par Amir et après avoir conclu que le viol déclenché par la victime (dans le sens utilisé par Wolfgang dans les cas d'homicide) n'existe pas, les auteurs admettent plus loin que certains cas de viol ne peuvent être expliqués adéquatement sans tenir compte du rôle joué par la victime:

Bien que nos données montrent que les victimes provoquent rarement sinon jamais une agression sexuelle (du moins, dans le sens retenu par Wolfgang), dans certains cas, ces victimes peuvent contribuer à la faciliter. Dans 9, 2\% des cas de Winnipeg, tant la victime que l'auteur estimaient ainsi que la victime avait été partiellement responsable de ce qui lui était arrivé. Ces cas incluaient des situations où, avant le viol, la victime avait aguiché l'agresseur, avait accepté d'avoir des rapports sexuels avec lui (et n'avait donc changé d'avis que plus tard), avait essayé d'être «attrapée» par lui, voire avait déjà volontairement retiré ses vêtements. Il n'est aucunement question ici de blâmer la victime. Une femme a bien sûr le droit de changer d'avis quant au fait d'avoir ou non des rapports sexuels, et son refus d'en avoir [même au dernier moment] ne doit jamais cautionner un viol. Toutefois, dans les termes de la perspective situationnelle qui sont les nôtres, la probabilité qu'un viol soit sélectionné parmi les options possibles augmente si la victime est (ou si elle pensait être) initialement sensible aux avances sexuelles de son futur agresseur. (Ibid.: 60 ; notre traduction)

\section{L'affrontement entre l'empirie et l'idéologie}

Une victimologie scientifique, apolitique et impartiale est une victimologie objective, neutre, orientée sur la recherche et centrée sur la théorie. C'est une discipline savante non idéologique qui ne dévie pas sur des jugements de valeur et qui ne prend pas position. C'est une victimologie qui accorde la même attention aux crimes commis contre des individus qu'aux crimes commis contre des groupes ou des populations entières. C'est une victimologie qui est tout aussi intéressée aux victimisations perpétrées par les puissants que par les victimisations commises par les démunis.

Une victimologie scientifique est une victimologie neutre sur le plan du genre, de l'origine ethnique et de la classe. C'est une victimologie égalitaire qui ne crée pas une hiérarchie normative de victimes, qui ne considère pas certaines victimes comme méritant plus d'attention que d'autres et qui n'offre pas de traitement préférentiel à certaines victimes ou à certains groupes de victimes. En d'autres mots, c'est une victimo- 
logie qui n'est ni constituée ni même affectée par des jugements de valeur.

La transformation idéologique de la victimologie a été nuisible à l'enrichissement et au progrès de la victimologie scientifique. Elle a créé un fossé presque infranchissable entre, d'une part, les victimologues portés par la science et, d'autre part, les victimologues guidés par l'action et engagés dans une idéologie. Leurs objectifs respectifs sont différents, leur langage est distinct et ils sont difficilement sur la même longueur d'onde. Laffrontement entre le scientifique et le politique, l'empirie et l'idéologie a été bien décrit par Donald Cressey dans une de ses dernières conférences, celle qu'il a présentée au cinquième Symposium international de victimologie tenu à Zagreb en août 1985 . Refusant de rejoindre les rangs de tous ceux qui évoquaient une régression malheureuse et une transformation de la victimologie vers un destin qui ne serait plus scientifique, Cressey déclara ouvertement que la victimologie n'était en fait ni une discipline scientifique ni même un champ académique (au contraire, par exemple, de la criminologie ou de l'écologie). Il y vit plutôt un programme non académique dans lequel un monceau d'idées, de centres d'intérêt, d'idéologies et de méthodes de recherche avaient été groupés de façon assez arbitraire. Cressey décrivit ainsi l'état de la victimologie en des termes relativement peu flatteurs:

Plus spécifiquement, la victimologie est caractérisée par un affrontement entre deux orientations également désirables concernant la souffrance humaine, l'une est humaniste, l'autre scientifique. Le travail des humanistes tend à être déprécié parce qu'il est considéré plus propagandiste que scientifique, et le travail scientifique tend à être déprécié parce qu'il n'est pas suffisamment orienté vers l'action sociale. (Cressey, 1985: 43; notre traduction)

Cressey notait qu'aux États-Unis au moins, une proportion considérable des victimologues étaient des activistes politiques et des travailleurs sociaux dont les intérêts premiers en victimologie étaient d'obtenir justice pour des personnes qui avaient été directement blessées (physiquement, économiquement ou psychologiquement) par des délinquants urbains. Il ajoutait quaucune recherche empirique n'est nécessaire pour soutenir la cause humanitaire et venir en aide aux blessés, incluant les victimes directes de crimes. Il affirmera aussi que la plupart des victimologues de «la loi et de l'ordre», à savoir ceux qui veulent durcir la 
réaction au crime afin de réduire les taux de victimisation du crime, sont des idéologues et non pas des scientifiques.

\section{La pauvreté théorique de la victimologie activiste}

Comme Cressey l'avait noté dans un article cité précédemment, «les victimologues humanistes sont intéressés à ériger un phénomène en problème social plutôt qu'à l'étudier scientifiquement» (1992: 69; notre traduction). Volontairement ou pas, consciemment ou non, les défenseurs et les lobbyistes des victimes jouent le jeu des politiciens conservateurs et néoconservateurs. Ils ont contribué à la propagation des idées, de la philosophie et des politiques de la criminologie de droite. Dans un tel climat, les recherches scientifiques s'intéressant aux interactions victime-agresseur ainsi qu'à la contribution de la victime à la genèse du crime en viennent à être qualifiées de tentatives de blâmer la victime et sont par conséquent écartées.

La transformation idéologique récente de la victimologie n'a pas été réalisée sans sérieuses conséquences négatives. Un des résultats a été le recentrement de la criminalité sur les crimes traditionnels, ceux qui connaissent une victime directe, immédiate, tangible et identifiable. Les crimes en col blanc, les crimes des entreprises et les actions causant d'importants dommages économiques et sociaux, peu importe qu'ils soient définis ou non comme des crimes, ont été une fois de plus relégués au second plan. Les activistes pro-victimes ont concentré leur attention et investi tous leurs efforts sur les crimes appelés «conventionnels» ou «de rue». La criminalité des entreprises et la criminalité d'affaires qui victimisent des millions et des millions de gens restent encore largement non rapportées et non poursuivies. En dépit de l'importance du crime en col blanc, pourtant largement responsable du quasi-effondrement récent de l'économie mondiale, et bien que ses préjudices excèdent de loin ceux causés par la délinquance en milieu urbain, ces crimes sont totalement occultés dans les campagnes de victimes. C'est aussi le cas pour d'autres actions socialement dommageables comme la pollution de l'environnement, la production de substances nocives, l'élimination de substances ou de produits chimiques dangereux, la fabrication de produits malsains, la violation des codes de sécurité et de la santé, pour n'en mentionner que quelques-uns, et sans compter les victimes d'abus du pouvoir politique et économique ou encore les victimes du terrorisme d'État. 
Peu importe que ce soit pour des raisons idéologiques, politiques ou pragmatiques, les mouvements de victimes ont été largement sélectifs et même discriminatoires dans leurs centres d'intérêt, leur insistance et leur action. Ils ont été très sélectifs tant dans les groupes de victimes qu'ils ont retenus que dans les types de crimes contre lesquels ils ont choisi de lutter. Le résultat, c'est qu'une grande majorité de victimes de crimes sont laissées sans protection, sans assistance et ne sont tout simplement pas entendues.

\section{Les fondations fragiles des politiques actuelles en matière de victimes}

Manifester un véritable intérêt pour soulager la souffrance humaine exige que la recherche et l'action soient en prise directe sur la compréhension, la réduction et la prévention de la victimisation, peu importe que cette dernière soit physique, sexuelle, mentale ou économique; qu'elle soit intentionnelle, qu'elle survienne à la suite d'une action délibérée, un accident, une imprudence, une insouciance ou une négligence; peu importe aussi qu'elle ait lieu à la maison, dans la rue ou au travail; peu importe enfin qu'elle soit le fait d'individus, d'organisations, d'entreprises ou encore du gouvernement lui-même.

Bien que portée par un objectif noble, la victimologie humaniste est pour l'essentiel non scientifique, dominée par une idéologie et gangrénée par l'air du temps. Elle laisse le politique envahir son territoire, alors que ce champ a tant souffert pour mériter une reconnaissance scientifique en approfondissant sa compréhension des dynamiques du crime. Les études de victimisation mises à part, la nouvelle victimologie a peu ajouté au savoir criminologique existant. Si la recherche sur la victimisation a conduit à certaines formulations théoriques intéressantes telles que le modèle de style de vie (voir Garofalo, 1986), la nouvelle tendance «appliquée» a entravé et ralenti le progrès de la théorie victimologique. Les postulats idéologiques ont remplacé les notions savantes. Les hypothèses et les tentatives pour mettre en lumière les dynamiques de la victimisation n'ont pas été débattues sur un plan scientifique mais bien sur un plan idéologique et philosophique.

Comme discipline émergente, la «vieille» victimologie avait ses failles et ses points faibles, mais son objectif était de fournir des fondations scientifiques à une théorie criminologique dynamique ainsi qu'une politique de prévention efficace centrée sur les victimes. La 
«nouvelle» victimologie appelle plutôt à davantage de mêmes remèdes inefficaces et de solutions dépassées. Un retour au "nœud coulant», aux longues sentences de prison, aux restrictions des remises en liberté sous caution et des libérations conditionnelles n'est pas susceptible de faire reculer le crime. Ces mesures n'ont pas été efficaces dans le passé et elles ne le seront pas davantage dans le futur. Elles détournent simplement l'attention et les fonds de ce qui pourrait et de ce qui devrait être fait pour changer les conditions socioéconomiques qui engendrent le crime et qui transforment certains individus en délinquants sans pitié ni remords. Pourtant, ces remèdes dépassés continuent à faire l'objet de demandes politiques de défenseurs des victimes, de comités officiels et autres commissions d'étude auxquels on demande de formuler des recommandations pour améliorer le sort des victimes de crimes. La commission d'étude du président Reagan (1982) recommandait par exemple l'abolition de la «règle d'exclusion» qui rendait inadmissible dans les procès criminels toute preuve recueillie illégalement. Elle recommandait aussi des peines de prison plus longues, l'abolition de la libération conditionnelle et la mise en ouvre d'une politique globalement plus dure envers les criminels urbains.

Des slogans comme «La justice pour les victimes» sont invariablement interprétés dans l'opinion publique comme signifiant plus de punition pour les délinquants. Un tel climat social ainsi créé pousse à perdre et à abandonner tout idéal de justice humaine, équitable et non rétributive. Les vieilles notions de châtiment et de rétribution sont au contraire ravivées, elles prennent de la vitesse en même temps qu'une nouvelle signification. Elles sont maintenant activement relancées, surtout ces quelques dernières décennies. Bien que les études manquent à ce sujet, il y a de fortes raisons de croire que la posture belliqueuse prise par les groupes de victimes a été largement ou partiellement responsable de la tendance nette vers des sanctions plus sévères, un plus large usage de l'emprisonnement et des durées plus longues d'incarcération. L'ironie, c'est que peu de victimes sont aidées en envoyant de plus en plus de délinquants en prison pour des durées toujours plus longues. L'argent qui pourrait être positivement dépensé à aider et à dédommager les victimes est ainsi gaspillé en incarcération improductive. Quant à la restitution par l'auteur de l'infraction, seul espoir de rétablir un semblant d'équilibre au profit des victimes de crimes de propriété qui n'étaient pas assurées, elle est entravée et non valorisée par l'incarcération du délinquant (Fattah, 1997a; 1997b). 


\section{Les dangers liés au zèle du missionnaire \\ Les conséquences non anticipées et indésirables d'une approche partiale et controversée}

Dans sa critique savante de la discipline de la gérontologie sociale, Smith (1989) avertit du danger du «zèle du missionnaire» déployé par des gérontologues sociaux au profit des intérêts des membres de la société qui sont plus âgés que les autres. Smith suggère que ce zèle du missionnaire peut facilement mettre en danger la "posture scientifique» du chercheur et dès lors sa contribution potentielle à des politiques sociales de recherche sur le vieil âge. Après avoir souligné les nombreux dangers que représente, pour les gérontologues, une sur-identification aux personnes âgées, Smith va recommander qu'on étudie de plus près la teneur de cette relation entre sur-identification et recherche savante dans le domaine de la gérontologie sociale.

Larticle de Smith permet donc de faire des parallèles frappants entre la situation en gérontologie sociale et les récentes transformations qui ont touché le champ de la victimologie. Sa mutation idéologique est manifeste dans les conférences et les symposia qui se tiennent en son nom: l'étude des victimes qui cède le pas à l'art de les aider, la suridentification avec des victimes de crimes, le zèle du missionnaire avec lequel les «intérêts» de la victime sont défendus et poursuivis, etc. Ceux qui sont soucieux d'une neutralité et d'une objectivité perdues de la discipline peuvent s'inquiéter d'être les témoins d'un glissement préoccupant: de savoir scientifique dépassionné, non biaisé et impartial, la victimologie est devenue un plaidoyer politique tombé dans un sectarisme déclaré (Fattah, 1992).

Le zèle du missionnaire montré par de nombreux victimologues au nom et dans l'intérêt des victimes de crimes est lourd de dangers. D'abord, et comme suggéré plus haut, il compromet la qualité de la connaissance et la position scientifique de la discipline victimologique. En conséquence, la victimologie est de plus en plus regardée comme un mouvement idéologique et humanitaire plutôt que comme une discipline scientifique (voir Cressey, 1992).

Le zèle du missionnaire et sa posture partisane orientent en outre le droit criminel et le système de justice pénale dans une direction punitive et rétributive. Mais ce n'est pas tout. Depuis que les groupes de pression des victimes ont choisi de mettre l'accent sur les crimes traditionnels plutôt que sur les crimes en col blanc ou les actes d'abus de pouvoir, la 
recherche a privilégié les études sur les premiers au détriment des seconds. Les victimes du crime en col blanc, du crime d'entreprise et de l'abus de pouvoir ont été une fois encore reléguées à l'arrière-plan.

Mais il y a encore un danger plus sérieux. Derrière la quête assoiffée des droits des victimes, on retrouve une volonté, manifeste ou latente, de sacrifier les droits des délinquants. Une fausse lutte est ainsi créée entre les droits des deux groupes. Karmen (1990) cite ceux qui affirment que les droits des victimes devraient être obtenus au détriment des droits des délinquants, qui déclarent que trop d'attention a été portée aux «droits des criminels» et pas assez à la détresse des personnes innocentes qu'ils ont blessées. Karmen donne le résumé suivant des requêtes de plaidoyers de victimes:

Pour restaurer une apparence d'équilibre sur l'échelle de la justice, qui a trop penché en faveur des criminels, certains des privilèges et des opportunités «anti-victimes» que les délinquants ont accumulés doivent être enlevés. Selon cette analyse, les victimes ont besoin de droits pour contrebalancer, égaler ou même «retourner» les droits des criminels. Dans ce contexte, la réforme signifie renverser les décisions précédentes des tribunaux et de manière générale les tendances juridiques antérieures, changer la balance du pouvoir afin que dorénavant, elle se fasse au détriment des contrevenants et donc au profit des parties blessées. (Karmen, 1990: 331; notre traduction)

Le rapport de la commission d'étude mandatée par le président Reagan (1982) et ses recommandations peuvent être clairement lus comme un réquisitoire accablant contre nombre de garanties juridiques que le système de justice criminelle étatsunien a établi au cours des années pour éviter la condamnation d'innocents et faire respecter les droits et libertés si chers à la démocratie (Fattah, 1992b: 13).

Il nous faut toutefois relever que l'accent mis sur les droits des victimes et l'insistance pour créer une lutte entre les droits des victimes et ceux des délinquants ont été beaucoup plus prononcés en Amérique du Nord qu'en Grande-Bretagne et dans bien d'autres pays. En retraçant l'historique du mouvement des victimes à la fois en Grande-Bretagne et aux États-Unis, Mawby et Gill (1987) ont conclu que même si le mouvement britannique avait obtenu un soutien majeur de ceux qui sont situés à droite du spectre politique, ce sont les besoins des victimes et la meilleure manière de les satisfaire qui sont toujours restés au centre des préoccupations. Par contraste, le mouvement des victimes aux États-Unis et au Canada s'est plus directement inquiété de la ques- 
tion de leurs droits. Rappelant combien il était simpliste de suggérer que les victimes recevaient beaucoup moins du système de justice criminelle que les contrevenants, Mawby et Gill ont opté pour une posture prudente et équilibrée. Ils affirment en effet que si les droits des victimes doivent être reconnus, cela doit être fait sans caricaturer l'ordre public étatique ou les fameux «privilèges» reconnus aux accusés et autres infracteurs.

\section{Quatrième partie : où va la « victimologie »?}

La crise actuelle de la victimologie: la stérilité scientifique du politique et de l'idéologie

À la première Conférence nationale sur les victimes de crime à Toronto en 1985, le mouvement des victimes a été reconnu comme le nouveau «succès industriel» de la décennie. En Grande-Bretagne, il a ainsi été considéré comme le mouvement à but non lucratif qui a connu l'expansion la plus rapide. On doit de fait admettre que, dans les trois dernières décennies, les groupes et les organisations de victimes ont poussé comme des champignons à travers toute l'Amérique du Nord, l'Europe et d'autres parties du monde. Inévitablement, cette croissance phénoménale a eu un effet significatif (de mon point de vue, négatif) sur la jeune discipline prometteuse qu'était alors la victimologie. Dans une grande mesure, les rencontres de victimologie ont cessé d'être des réunions savantes où les découvertes de la recherche scientifique sur les victimes sont présentées et discutées. Elles sont plutôt devenues un forum pour la rhétorique politique et idéologique. Elles se sont traduites par une transformation de la victimologie, passée de discipline scientifique orientée vers la recherche à un mouvement humaniste, glissant de la recherche savante à l'activisme politique. De sorte que la meilleure manière de décrire la victimologie aujourd'hui, c'est de la définir comme un mouvement idéologique politique et une profession d'aide, pas très différente finalement des soins infirmiers ou du travail social.

Les campagnes politiques et les groupes de pression au nom des victimes de crimes, même quand ils sont motivés par le plus noble des soucis humanitaires pour le bien-être des victimes, n'ont aucun rapport avec la science. La «recherche» victimologique conduite par les victimologues activistes et lobbyistes est aussi objective que la recherche soutenue par le lobby des armes à feu sur l'enjeu du contrôle des armes. Les groupes de pression, par nature ou par choix, ne disposent pas de 
la neutralité et de l'impartialité nécessaires à l'établissement d'une érudition solide, objective et désintéressée. L'activisme politique et la connaissance savante dépassionnée ne vont pas de pair. Nous ne faisons pas ici un plaidoyer idéaliste qui encouragerait une tour d'ivoire et une passivité académiques, mais plutôt un appel pour séparer la science de la politique, et pour distinguer le rôle du scientifique de celui du lobbyiste.

\section{L'avenir incertain de la victimologie}

Où va donc la «victimologie»? Se dirige-t-on vers un affrontement entre humanistes et scientifiques? Cet affrontement est-il inévitable? Pourrait-il être évité? Un tel affrontement signerait-il la fin de la victimologie comme discipline scientifique? Ce sont toutes des questions difficiles, et les réponses ne peuvent pour le moment qu'être spéculatives. Cressey (1985) suggère une façon d'atténuer l'affrontement potentiel entre humanistes et scientifiques. Il estime que tous devraient être encouragés à comprendre que la victimologie est d'abord une entreprise de recherche scientifique et que l'association des victimologues est d'abord une association de chercheurs. Cette définition, il le reconnait, exclurait les humanistes mais ils pourraient facilement trouver «refuge» dans des associations pour les droits humains. Quant à ceux qui sont engagés dans l'action, ils pourraient travailler aux côtés des travailleurs sociaux:

Dans le cas contraire, la victimologie pourrait s'éteindre. Et si elle prend cette voie-là, les victimologues humanistes pourraient chercher une alliance avec d'autres champions des droits humains pendant que les victimologues scientifiques pourraient finir par rejoindre leurs collègues des sciences sociales qui s'appellent eux-mêmes criminologues. Si l'objectif de la victimologie se limite aux victimes de crimes, il n'y a de fait plus de raison convaincante de la maintenir séparée de la criminologie. (Cressey, 1985: 54 ; notre traduction)

\section{Conclusion}

La science et la partisanerie sont incompatibles. Une fois que les chercheurs prennent position ou deviennent militants, ils perdent leur neutralité, leur objectivité et leur crédibilité. C'est un principe fondamental qui devrait être sérieusement pris en compte par les criminolo- 
gues et les victimologues bien intentionnés qui ont adopté la cause des victimes de crimes et qui affirment parler en leur nom. C'est aussi un principe qui va radicalement à l'encontre de la posture des défenseurs des droits des victimes qui tentent continuellement de séparer la victimologie de la criminologie et qui essaient de créer une entreprise politique et idéologique qui n'a rien à voir avec la science (Fattah, 2008).

L'avenir de la victimologie dépendra ainsi de sa capacité à revenir à sa mission scientifique originale, à se débarrasser de son manteau idéologique et à reprendre son rôle de discipline savante comme part intégrante de la criminologie. C'est la nécessité de séparer la recherche de l'action et la science de l'activisme qui dicte que la victimologie doit être séparée des politiques en matière de victimes. Pour restaurer la neutralité de la victimologie et regagner et maintenir son intégrité scientifique, elle doit se détacher du politique et de l'idéologie. C'était précisément le besoin d'affirmer le caractère non normatif et non idéologique de la criminologie qui a conduit certains des plus fameux criminologues du $\mathrm{xx}^{\mathrm{e}}$ siècle, tels que Thorsten Sellin (1938) et Hermann Mannheim (1965), pour n'en citer que quelques-uns, à appeler au divorce des politiques criminelles et de la criminologie.

Dès 1938, Sellin suggérait que le terme «criminologie» ne soit utilisé que pour désigner le corps des connaissances scientifiques ainsi que la poursuite délibérée d'un tel savoir. Il proposa alors que l'usage technique du savoir dans le traitement et la prévention du crime soit séparé de la «criminologie». Comme il ne parvenait pas à trouver un terme adéquat pour désigner ce champ, il finit par retenir une notion qui ne le satisfaisait pas vraiment, celle de «technologie criminelle» (crimino-technology).

Trois décennies plus tard, Hermann Mannheim (1965: 13) suggéra lui aussi que la politique criminelle soit traitée comme une discipline à part, plutôt qu'à titre de partie intégrante de la criminologie. Affirmant le caractère non normatif de la criminologie, Mannheim pensait qu'il était préférable que les questions se rapportant à ce qui aurait d̂ิ être fait pour réformer le droit criminel et le système pénal soient traitées dans une discipline séparée, fondée sur les découvertes factuelles des criminologues et des pénologues. La criminologie, insista-t-il, devrait rester une discipline non versée dans l'élaboration de décisions politiques, une discipline qui regarde les «fins» au-delà de sa province. Mannheim s'empressa d'ajouter que cela n'empêchait pas pour autant le criminologue de promouvoir des projets de loi en vue de réformes 
juridiques, pénales ou administratives, mais qu'il devait alors assurer cette promotion à titre de politicien ou de citoyen ordinaire et non en tant que criminologue.

En accord avec leurs réflexions, je suggère que le terme «victimologie» ne soit utilisé que pour désigner le corps de la connaissance scientifique portant sur les victimes ainsi que la poursuite d'un tel savoir. Le conseil que Mannheim (1965) donnait aux criminologues s'applique de la même façon aux victimologues. La victimologie, répétons-le, devrait rester une entreprise savante et scientifique, une discipline non versée dans l'élaboration de décisions politiques, qui regarde les «fins» au-delà de sa province. Je ne peux m'empêcher d'ajouter que la sagesse d'un tel conseil n'a jamais été aussi évidente qu'aujourd'hui.

Un fait qui est souvent ignoré ou négligé dans les sciences sociales, c'est que l'édification d'un savoir et sa mise en œuvre, son application pratique, sont deux entreprises différentes, distinctes et séparées. La quête du savoir pour le savoir, comme c'est le cas en anthropologie, en archéologie ou en histoire, est un exercice louable qui en vaut la peine, peu importe que ce savoir ait ou non des applications pratiques. C'est une quête d'autant plus précieuse qu'elle est neutre et qu'elle ne court pas le danger de voir les connaissances acquises détournées ou déformées une fois appliquées (Fattah, 2008).

\section{Références}

Amir, M. (1971). Patterns in Forcible Rape. Chicago: University of Chicago Press. Block, R. (1977). Violent Crime. Lexington, MA: D. C. Heath \& Co.

Block, R., \& Skogan, W. G. (1986). Resistance and Non-fatal Outcomes in Stranger to Stranger Predatory Crime. Violence and Victims, 1 (4), 241-253.

Chambers, G., \& Tombs, J. (Eds.) (1984). The British Crime Survey Scotland. A Scottish Office Social Research Study. Edinburgh: Her Majesty's Stationary Office.

Chappell, D., \& James, J. (1986). Victim Selection and Apprehension from the Rapist's Perspective: A Preliminary Investigation. In K. Miyazawa \& M. Ohya (Eds.), Victimology in Comparative Perspective (tiré à part). Tokyo: Seibundo Publishing Co. Ltd.

Clark, L., \& Lewis, D. (1977). Rape: The Price of Coercive Sexuality. Toronto: The Women's Press.

Cook, P. J. (1986). The Relationship between Victim Resistance and Injury in Non-commercial Robbery. Journal of Legal Studies. XV, 405-416.

Cressey, D. R. (1985). Research implications of conflicting conceptions of victimology. In Z. P. Separovic (Ed.), International action and study of victims 
(43-54). Zagreb: University of Zagreb. Reprinted in 1992 In E. A. Fattah (Ed.), Towards a Critical Victimology (57-73). London: Macmillan.

Curtis, L. (1974). Criminal Violence: National Patterns and Behaviour. Lexington, MA: D. C. Heath \& Co.

Del Frate, A. A., Zvecic, I., \& Van Dijk, J. J. M. (1993). Understanding Crime Experiences of Crime and Crime Control. Rome: UNICRI.

Ellenberger, H. (1955). Psychological Relationships between the Criminal and his Victim. Archives of Criminal Psychodynamics, 2, 257-290.

Fattah, E. A. (1967a). La victimologie: qu'est-elle et quel est son avenir? (Première partie). Revue internationale de criminologie et de police technique, 21 (2), 113-124.

(1967b). La victimologie: qu'est-elle et quel est son avenir? (Suite et fin). Revue internationale de criminologie et de police technique, 21 (3), 192-202.

- (1973). Le rôle de la victime dans le passage à l'acte. Revue internationale de criminologie et de police technique, 36 (2), 173-188.

- (1976). The use of the victim as an agent of self-legitimization: Towards a dynamic explanation of criminal behavior. In E. C. Viano (Ed.), Victims and society (105-129). Washington, DC: Visage Press.

(1979). Some recent theoretical developments in victimology. Victimology: An International Journal, 4, 198-213.

(1980a). Some Reflections on the Victimology of Terrorism. Terrorism: An International Journal, 3 (2), 81-108.

- (1980b). Victimologie: tendances récentes. Criminologie, 13 (1), 6-36.

(1981a). Becoming a Victim: The Victimization Experience and its Aftermath. Victimology: An International Journal, 6 (1-4), 29-47.

- (1981b). La victimologie entre les attaques idéologiques et les critiques épistémologiques. Déviance et Société, $V(1), 71-92$.

- - (1984). Victims' response to confrontational victimization: A neglected aspect of victim research. Crime and Delinquency, 30 (1), 75-89.

(1986). Victimología: tendencias recientes. Revista Mexicana de Justicia, 20 (mai), 43-79.

- (1988). Some Recent Theoretical Developments in Victimology. Proceedings of the $8^{\text {th }}$ International Congress of Criminology (659-693). Lisbonne, Portugal: Ministère de la Justice.

(1989). Victims and Victimology: The Facts and the Rhetoric. International Review of Victimology, 1 (1), 1-21.

- _ (1991a). Understanding criminal victimization. Scarborough, Ontario: Prentice Hall.

- (1991b). From crime policy to victim policy - The need for a fundamental change. International Annals of Criminology, 29, $1 \& 2$.

(Ed.) (1992a). Towards a critical victimology. London, UK: Macmillan/New York, NY: St. Martin's Press.

(1992b). The need for a critical victimology. In E. A. Fattah (Ed.), Towards a Critical Victimology (3-26). London, UK: Macmillan / New York, NY: St. Martin's Press. 
(1992c). Victims and victimology: The facts and the rhetoric. In E. A. Fattah (Ed.), Towards a critical victimology (29-56). London, UK: Macmillan/ New York, NY: St. Martin's Press.

(1992d). The UN declaration of basic principles of justice for victims of crime and abuse of power: A constructive critique. In E. A. Fattah (Ed.), Towards a critical victimology (401-424). London, UK: Macmillan/New York, NY : St. Martin's Press.

-_ (1993a). La relativité culturelle de la victimisation. Criminologie, 26 (2), 121-136.

-_ (1993b). Doing unto others: The revolving roles of victim and victimizer. Simon Fraser University Alumni Journal, 11 (1), 12-15.

(1994a). The interchangeable roles of victim and victimizer. Second Inkeri Anttila's Honour Lecture. Helsinki, Finland: HEUNI. Traduit vers l'espagnol (Los roles intercambiables de víctima y victimario) et publié in 1997, Cuadernos de criminología, 7, 23-54.

-_ (1994b). La victimologie au carrefour: entre la science et l'idéologie. Présentations à la Société Royale du Canada, 47, 159-72.

- _ (1994c). Some recent theoretical developments in victimology. In P. Rock (Ed.), Victimology (285-300). Dartmouth: Aldershot.

-_ (1994d). Victimology: Some problematic concepts, unjustified criticism and popular misconceptions. In G. F. Kirchhoff, E. Kosovski \& H. J. Schneider (Eds.), International Debates of Victimology (82-103). Mönchengladbach: WSP.

- _ (1995). La victimologie au carrefour : entre la science et l'idéologie. Revue internationale de criminologie et de police technique, 2, 131-39.

-_ (1997a). Criminology: Past, Present and Future - A Critical Overview. London, UK: Macmillan / New York, NY: St. Martin's Press.

(1997b). Los roles intercambiables de víctima y victimario. Cuadernos de criminologia, 7, 23-54.

- — (1997c). Víctimas y victimología: los hechos y la retórica. Victimología, $14,13-44$.

- - (1999). From a handful of dollars to tea and sympathy: The sad history of victim assistance. In J. J. M. Van Dijk, R. G. H. Van Kaam \& J. Wemmers (Eds.), Caring for crime victims: Selected proceedings of the $9^{\text {th }}$ international symposium on victimology (187-206). Monsey, NY: Criminal Justice.

-_ (2000a). Victimology: Past, Present and Future. Criminologie, 33 (1), 17-46.

- - (2000b). Victimology Today: Recent Theoretical and Applied Developments. Resource Material Series, 56, 60-70. Fuchu/Tokyo: UNAFEI.

- (2001). Preventing Repeat Victimization as the Ultimate Goal of Victim Services. International Annals of Criminology, 38 (1/2): 113-33.

- - (2004). Positions savantes et idéologiques sur le rôle de la victime et sa contribution à la genèse du crime. In R. Cario \& P. Mbanzoulou (Eds.), La victime est-elle coupable? (23-41). Paris: L'Harmattan. 
(2008). The future of criminology as a social science and academic discipline: Reflections on criminology's unholy alliance with criminal policy $\&$ on current attempts to divorce victimology from criminology. International Annals of Criminologie, 45 (1\&2), 135-170.

(2010). The Evolution of a Young, Promising Discipline: Sixty Years of Victimology, A Retrospective and Prospective Look. In P. Knepper, S. Shoham \& M. Kett (Eds.), International Handbook of Victimology (43-94). Boca Raton/London/New York: Taylor and Francis.

Felson, R. B., \& Steadman, H. J. (1983). Situational Factors in Disputes Leading to Criminal Violence. Criminology, 21 (1) 59-74.

Franklin II, C. W., \& Franklin, A. P. (1976). Victimology Revisited: A Critique and Suggestions for Future Direction. Criminology, 14 (1), 177-214.

Gibson, L., Linden, R., \& Johnson, S. (1980). A Situational Theory of Rape. Canadian Journal of Criminology, 22 (1), 51-65.

Gottfredson, M. R. (1984). Victims of crime: The dimensions of risk. Home Office Research and Planning Unit Report No. 81. London: HMSO.

Hatalak, O., Del Frate, A. A., \& Zvekic, U. (1998). The International Crime Victim Survey in Countries in Transition. Rome: UNICRI.

Hepworth, M. (1975). Blackmail: Publicity and Secrecy in Everyday Life. London: Routledge \& Kegan.

Hindelang, M., Gottfredson, M., \& Garofalo, J. (1978). Victims of Personal Crime. Cambridge, MA: Ballinger.

Hinrichs, R. (1987). Das Chronische Opfer. Stuttgart: Georg Thieme Verlag.

Karmen, A. (1990). Crime Victims: An Introduction to Victimology ( $2^{\text {nd }}$ ed.). Monterey: Brooks/Cole.

- (1996). Crime Victims: An Introduction to Victimology (3 $3^{\text {rd }}$ ed.). Belmont, CA: Wadsworth.

Mannheim, H. (1965). Comparative Criminology. London: Rutledge and Kegan.

Normandeau, A. (1968). Trends and Patterns in Crimes of Robbery. Ph.D. Dissertation. Philadelphia: University of Pennsylvania.

Padowetz, M. (1954). Der Heiratsschwindel. Wien: Springer.

Pittman, D. J., \& Handy, W. (1964). Patterns in Criminal Aggravated Assault. Journal of Criminal Law, Criminology and Police Science, 55 (1), 462-469.

Rock, P. (1990). Helping crime victims: The home office and the rise of victim support in England and Wales. Oxford, UK: Clarendon.

- - (1994). Victimology. Aldershot: Dartmouth.

Sellin, T. (1938). Culture Conflit and Crime. New York: Social Science Research Council.

Silverman, R. (1973). Victim Precipitation: An Examination of the Concept. In I. Drapkin \& E. Viano (Eds.), Victimology: A New Focus (vol. 1). Lexington, MA.: D. C. Heath \& Co.

Singer, S. (1981). Homogeneous victim-offender populations: A review and some research implications. Journal of Criminal Law and Criminology, 72, 779-88. 
Smith, G. (1989). Missionary Zeal and the Scholarly Stance: Policy and Commitment in Research on Old Age. Ageing and Society, 9, 105-121.

Smith, S. J. (1986). Crime, space and society. Cambridge: Cambridge University Press.

Sparks, R. F., Genn, H., \& Dodd, D. (1977). Surveying Victims: A Study of the Measurement of Criminal Victimization. London: John Wiley and Sons.

Sparks, R. F. (1981). Multiple Victimization: Evidence, Theory and Future Research. The Journal of Criminal Law, Criminology and police Science, 72, 762778.

Sutherland, E., \& Cressey, D. (1960). Principles of Criminology (6 ${ }^{\text {th }}$ edition). New York: Lippincott.

Timmer, D., \& Norman, W. H. (1984). The Ideology of Victim Precipitation. Criminal Justice Review, 9, 63-68.

United Nations (1985). Declaration of basic principles of justice for victims of crime and abuse of power. New York: United Nations' Department of Public Information.

United States of America (1982). President's task force on victims of crime: Report. Washington, DC: Government Printing Office.

United States of America, Bureau of Justice Statistics (1987). Robbery Victims: Special Report. Washington, DC: Government Printing Office.

Van Dijk, J. J. M., Mayhew, P., \& Killias, M. (1990). Experiences of Crime across the World - Key Findings of the 1989 International Crime Survey. Deventer: Kluwer Law and Taxation Publishers.

Virkkunen, M. (1975). Victim-Precipitated Pedophilia Offences. British Journal of Criminology, 15 (2), 175-180.

Von Hentig, H. (1940/41). Remarks on the Interactions of Perpetrator and Victim. Journal of Criminal Law and Criminology, 31, 303-309.

- - (1948). The Criminal and his Victim. New Haven: Yale University Press.

Wertham, F. (1949). The Show of Violence. New York: Doubleday.

Wolfgang, M. E. (1957). Victim-precipitated criminal homicide. Journal of Criminal Law, Criminology and Police Science, 48 (1), 1-11.

- - (1958). Patterns in criminal homicide. Philadelphia : University of Pennsylvania Press.

- - (1982). Victim Intimidation, Resistance and Injury: A Study of Robbery. In K. Miyazawa \& M. Ohya (Eds.), Victimology in Comparative Perspective. Tokyo: Seibundo Publishing Co. Ltd.

Wright, R. (1980). Rape and Physical Violence. In D. J. West (Ed.), Sex Offenders in the Criminal Justice System (100-113). Cambridge: Institute of Criminology Series.

Ziegenhagen, E. A., \& Brosnan, D. (1985). Victim Responses to Robbery and Crime Control Policy. Criminology, 23 (4), 675-695.

Zimring, F. (1977). Determinants of the Death Rate of Robbery: A Detroit Time Study. Journal of Legal Studies, 6 (2), 317-332. 
Zimring, F., \& Zuehl, J. (1986). Victim Injury and Death in Urban Robbery: A Chicago Study. Journal of Legal Studies, XV, 1-31.

ABSTRACT - Victimology, the study of the victim, emerged in the second half of the $20^{\text {th }}$ century as a branch of criminology. Until then criminology was exclusively focused on crime and its perpetrators. But since most crimes are committed against a victim/ target the study of the latter offered a holistic approach. It also offered the prospect of transforming the static criminological theories into dynamic theories incorporating the interactions between victim and victimizer and the situational dynamics in confrontational victimizations. The beginnings of Victimology were purely theoretical focusing on the victims of specific crimes, their role and their eventual contribution to the genesis of the crime.

In the 1970's the micro approach that characterized early Victimology was eclipsed by a macro approach aimed at assessing the volume of victimization, particularly hidden and unreported victimization. Victimization surveys became quite popular and were carried out regionally, nationally and transnationally. They allowed researchers to collect a vast amount of data on crime victims and yielded some very interesting as well as some unexpected findings. The last decades of the 20th century witnessed a major transformation in Victimology. The Victimology of the act gave way to a Victimology of action. The ideological transformation of victimology from the study of the victim into the art of helping victims, the over-identification with crime victims, and the missionary zeal with which the 'interests' of those victims are defended and pursued are quite manifest in victimology conferences and symposia.

The missionary zeal exhibited by many victimologists on behalf and in the interest of crime victims is fraught with danger. First, it is jeopardizing the quality of scholarship and the scholarly stance of the discipline of victimology. As a result, victimology is increasingly being regarded as a humanitarian and ideological movement rather than a scientific discipline. Secondly, missionary zeal and partisan stance are moving criminal law and the criminal justice system into a punitive, retributive direction. There is also a third danger. Since the victim lobby has chosen to focus on traditional crimes rather than white-collar crime or acts of abuse of power, there has been a distinct shift of focus in research to the former type at the expense of the latter. Victims of whitecollar crime, corporate crime and abuse of power have once again been relegated to the shadow. More serious still is yet another danger. In the diligent quest for victims' rights there seems to be a manifest or latent willingness to sacrifice offenders' rights. A false contest is thus created between the rights of both groups.

So where is victimology heading? Science and partisanship are incompatible. Once researchers take sides or become advocates they lose their neutrality, their objectivity and their credibility. This is a fundamental principle that should be seriously considered by those well-intentioned criminologists and victimologists who have adopted the cause of crime victims and who claim to speak on their behalf.

The future of victimology will thus depend on its ability to return back to its original scientific mission, to shed its ideological mantle and to resume its role as a scholarly discipline and as an integral part of criminology. It is the need to separate research from action and science from activism that dictates that victimology be 
separated from victim policy. To restore its neutrality and to regain and maintain its scientific integrity victimology will have to detach itself from politics and ideology.

KEYWORDS - Victimology, activist victimology, victimization, victim surveys, confrontational victimization, victim precipitation, recidivist victim.

RESUMEN - Populares al inicio de la victimología, los estudios individuales de víctimas de crímenes específicos fueron eclipsados en los años 1970 por investigaciones sobre victimización a gran escala: la perspectiva micro dejó su lugar al enfoque macro. El objetivo principal de estas investigaciones fue determinar el volumen de victimización, identificar la población fuente y establecer las características sociodemográficas de las víctimas de crímenes. Cada una de ellas constituye una mina de información sobre las víctimas y permite el análisis minucioso de modelos y de tendencias espaciotemporales para diversos tipos de victimización.

En los tres últimos decenios del siglo xx, la victimología pasó por una metamorfosis tan importante como inquietante: su transformación ideológica se ha convertido en un obstáculo para el enriquecimiento y el avance científicos. La mutación ideológica de la victimología se manifiesta en las conferencias y simposios que se realizan en su nombre: el estudio de las víctimas cede su lugar al arte de ayudarlas, la identificación excesiva con las víctimas de crímenes y el celo de misionero con el que los "intereses" de la víctima se defienden, persiguen, etc. Todo lo cual señala un deslizamiento preocupante: de saber científico desapasionado, objetivo e imparcial, la victimología se ha convertido en un alegato político en ocasiones de un sectarismo declarado. El celo de misionero mostrado por algunos victimólogos en nombre del interés de las víctimas plantea diversos riesgos, algunos de los cuales se analizan en este artículo.

PALABRAS CLAVE - Victimología, victimología activista, victimización, encuestas de victimización, victimización confrontacional, víctima catalizadora, víctima reincidente. 